\title{
Atypical hemolytic-uremic syndrome
}

INSERM

\section{Source}

INSERM. (1999). Orphanet: an online rare disease and orphan drug data base. Atypical hemolytic-uremic syndrome. ORPHA:2134

Atypical hemolytic-uremic syndrome (aHUS) is a thrombotic microangiopathy characterized by mechanical hemolytic anemia, thrombocytopenia, and renal dysfunction. 